\title{
Supercontinuum Generation Assisted by Wave Trapping in Dispersion-Managed Integrated Silicon Waveguides
}

\author{
Junxiong Wei®, ${ }^{1,3}$ Charles Ciret, ${ }^{2}$ Maximilien Billet $\odot,{ }^{1,3}$ François Leo, ${ }^{1}$ Bart Kuyken, ${ }^{3}$ and \\ Simon-Pierre Gorza ${ }^{1, *}$ \\ ${ }^{1}$ OPERA-Photonique, Université libre de Bruxelles (ULB), Avenue Franklin D. Roosevelt 50, Bruxelles B-1050, \\ Belgium \\ ${ }^{2}$ Laboratoire de Photonique d'Angers EA 4464, Université d'Angers, 2 Boulevard Lavoisier, Angers 49000, \\ France \\ ${ }^{3}$ Department of Information Technology, Photonics Research Group, Ghent University -IMEC, Ghent B-9000, \\ Belgium
}

(Received 27 July 2020; revised 20 September 2020; accepted 13 October 2020; published 18 November 2020)

\begin{abstract}
Compact chip-scale comb sources are of significant interest for many practical applications. Here, we experimentally study the generation of supercontinuum (SC) in an axially varying integrated waveguide. We show that the local tuning of the dispersion enables the continuous blue shift of dispersive waves by more than $200 \mathrm{~nm}$ due to their trapping by the strongly compressed pump pulse. This mechanism provides an insight into supercontinuum generation in varying-dispersion integrated waveguides. Pumped close to $2.2 \mu \mathrm{m}$ in the femtosecond regime and at a pulse energy of approximately $4 \mathrm{pJ}$, the output spectrum extends from $1.1 \mu \mathrm{m}$ up to $2.76 \mu \mathrm{m}$ and shows good coherence properties. Octave-spanning SC is also observed at an input energy as low as approximately $0.9 \mathrm{pJ}$. We show that the supercontinuum is more robust against variations of the input-pulse parameters and is also spectrally flatter in waveguides with an optimized dispersion profile than in dispersion-engineered ones. This research demonstrates the potential of varying-dispersion waveguides for coherent SC generation and paves the way for integrated low-power applications, such as chip-scale frequency-comb generation, precision spectroscopy, optical-frequency metrology, and wide-band wavelength division multiplexing in the near infrared.
\end{abstract}

DOI: 10.1103/PhysRevApplied.14.054045

\section{INTRODUCTION}

Spectral broadening of light and the generation of new frequency components are ultimate features of nonlinear optics. They have been intensively studied since the discovery of second-harmonic generation in 1961 [1]. The particular nonlinear process known as supercontinuum (SC) generation occurs when narrow-band incident pulses or continuous waves experience a dramatic spectral broadening [2]. It was reported by Alfano and Shapiro in 1970 [3]. Since then, it has been observed in a wide variety of bulk nonlinear media [4-8] and various types of waveguides [9-11]. Today, broadband SC generation has found numerous applications, such as high-precision spectroscopy [12-14], frequency metrology and synthesis [15-17], ultrashort-pulse generation [18], wavelengthdivision-multiplexing (WDM) communication [19], and nonlinear optical spectroscopy and imaging in biophotonics [20-22]. The integration of SC sources on a chip, based on compact planar waveguide circuits rather than optical

\footnotetext{
*simon.pierre.gorza@ulb.be
}

fibers, can provide compact, robust, and power-efficient platforms for the aforementioned applications.

Both experimental and theoretical investigations of SC generation in waveguides have been a hot topic [23-28]. In order to build integrated electronic and photonic devices on the same chip for mass-market applications, optical waveguides should be fabricated together with silicon electronic components or be CMOS compatible. The siliconon-insulator (SOI) platform, which shows low propagation loss and high index contrast, is one of the leading candidates to achieve this purpose. Silicon also has a large nonlinear coefficient, similar to that of chalcogenide glass [29] and about 200 times higher than that of silica [30], facilitating ultracompact and power-efficient nonlinear devices for SC generation. In 2007, Yin et al. [31] reported numerical simulations of SC generation in a 1.2$\mathrm{cm}$-long crystalline silicon waveguide and predicted spectral broadening over $400 \mathrm{~nm}$. This was quickly followed by experiments with a 100 -fs pulse at $1.3 \mu \mathrm{m}$, showing spectral broadening of approximately $300 \mathrm{~nm}$ [32]. Later, SC generation pumped at telecommunication wavelengths and spanning from 1200 to $1700 \mathrm{~nm}$ in a standard (220 $\mathrm{nm}$ high) SOI waveguide was reported [33]. In both 
cases, the two-photon absorption clamps the maximum power in the chip, thereby impeding the maximum achievable spectral broadening. However, the nonlinear loss can be dramatically reduced when the pump wavelength lies beyond approximately $2200 \mathrm{~nm}$ [34-36]. Octave-spanning SC on a SOI chip, resorting to input pulses near $2.5 \mu \mathrm{m}$ and a 320 -nm- by 1210 -nm-cross-section waveguide, has been reported in Ref. [37]. In 2018, an octave-spanning $\mathrm{SC}$, covering 1.124-2.4 $\mu \mathrm{m}$, was generated in SOI ridge waveguides [38]. In order to avoid the inherent nonlinear loss encountered in silicon in the near infrared and achieve broader SC spectra, the research moved toward other platforms, such as silicon nitride [39], chalcogenide [40], silicon-germanium [41], indium gallium phosphide [42], and aluminum nitride [43]. In the context of SC generation, Guo et al. [44] have reported a mid-infrared comb source from $2.5 \mu \mathrm{m}$ to $4 \mu \mathrm{m}$ in silicon nitride $\left(\mathrm{Si}_{3} \mathrm{~N}_{4}\right)$ waveguides and, recently, a (400-2400)-nm supercontinuum source has been demonstrated in integrated lithium niobate waveguides [45].

It is now well understood that the dynamics of onchip broadband SC generation pumped in the anomalous dispersion regime with ultrashort pulses are dominated by self-phase modulation and temporal pulse compression, followed by higher-order soliton fission and emission of resonant dispersive waves [31,46]. In the absence of a Raman-induced self-frequency shift, the widespread strategy adopted to generate large spectra in these platforms merely consists in designing the cross section of the waveguide so as for the dispersive waves to be emitted as far as possible from the pump wavelength. In the literature, these structures are referred to as dispersion-engineered waveguides. Inspired by previous works with optical fibers (see, e.g., [47-51]), cascaded waveguides, tapers, and more complex dispersion-managed (DM) integrated structures have recently been investigated, both numerically $[52,53]$ and experimentally [54-56]. The motivation for studying varying-dispersion waveguides is the ability to locally adapt the phase-matching conditions required for the generation of new frequencies through four-wave mixing processes. This encompasses the generation of dispersive waves at different detunings from the pump wavelength but also frequency shifting through collisions and trapping at event horizons. The second advantage is the possibility of ensuring a high degree of coherence over the whole output spectrum by maintaining, at the beginning of the waveguide, a large anomalous dispersion or, equivalently, a low soliton order.

In this paper, we experimentally investigate the ability of DM designs to overcome the SC-generation performances of fixed-width (FW) and single-tapered (ST) nanophotonic waveguides. Three different dispersion maps (DM, ST, and FW) are optimally designed by numerical simulations of the nonlinear pulse propagation. Unlike previous works on SC generation in integrated waveguides with variable dispersion, our approach is not focused, $a$ priori, on a particular physical mechanism, such as multiple phase matching of dispersive waves or a short propagation distance in the anomalous dispersion regime. More complex dispersion profiles are also investigated. New or unexpected dynamics could thus be uncovered.

\section{EXPERIMENT}

\section{A. Method}

The experimental setup for SC generation is shown in Fig. 1. The frequency-comb seed source consists of a Kerr-lens mode-locked Cr:ZnSe femtosecond laser (MODEL:CLPF-2200-10-70-0.8, IPG). The output wavelength of the laser can be tuned from $2130 \mathrm{~nm}$ to 2260 $\mathrm{nm}$, close to the two-photon absorption band edge of the silicon at $2200 \mathrm{~nm}$. The femtosecond laser provides a maximum average power of $1.2 \mathrm{~W}$, with 70 -fs (fullwidth-at-half-maximum) pulses at a $82 \mathrm{MHz}$ repetition rate. A combination of a half-wave plate and a polarizer is used as a variable power attenuator and sets the polarization to excite the quasitransverse electric mode of the waveguide. The light is coupled into the waveguide by means of a microscope objective $(\times 60, \mathrm{NA}=0.65)$ and is collected by a silica-based lensed fiber (working distance $14 \mu \mathrm{m}, \mathrm{NA}=0.4$ ). This asymmetric coupling scheme ensures a close-to-transform-limited input pulse and a large output collection efficiency. The lensed-fiberto-waveguide coupling efficiency is estimated at $8.3 \mathrm{~dB}$ at the pump wavelength from measurements at low power with symmetric in- and out-couplings with lensed fibers. A coupling efficiency of $24.5 \mathrm{~dB}$ through the microscope objective is obtained from linear transmission experiments. As the collected power does not vary significantly between the waveguides, the in- and out-coupling efficiencies are assumed constant for the different experiments. The output

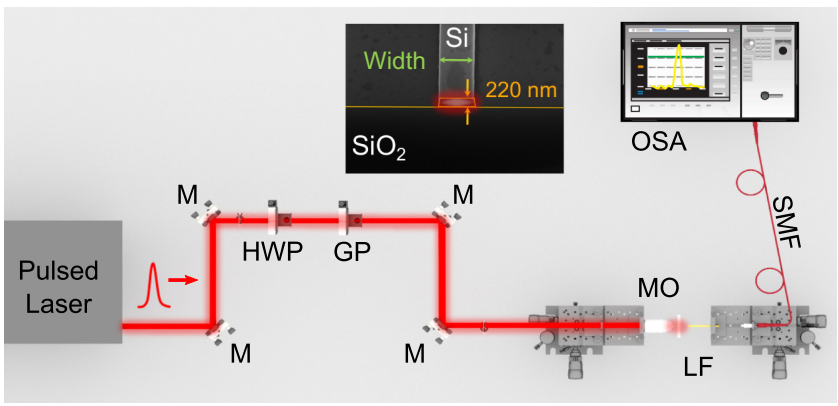

FIG. 1. The experimental setup for studying the SC generation in the nanophotonic waveguides (M, mirror; HWP, half wave plate; GP, Glan polarizer; MO, microscope objective; LF, lensed fiber; SMF, single-mode fiber; OSA, optical spectrum analyzer). The inset shows the scanning-electron-microscope image of the cross section of the silicon-on-silica waveguide. 
spectra are recorded using two optical-spectrum analyzers Yokogawa-AQ6370D (800-1700 nm) and AQ6376 $(1500-3400 \mathrm{~nm})$ to cover the entire supercontinuum bandwidth.

The air-clad SOI waveguides, fabricated in a CMOS pilot line, consist of a 220-nm-thick silicon layer on top of a $2-\mu \mathrm{m}$ buried oxide layer. The inset in Fig. 1 shows a scanning-electron-microscopy image of the cross section of the silicon wire. For SOI nanophotonic waveguides, the normal dispersion of bulk silicon can be compensated by the geometric waveguide dispersion. In 220-nm-thick structures, the group-velocity dispersion (GVD) depends strongly on the waveguide width and a net anomalous dispersion can be reached in the wavelength range of $1500-2300 \mathrm{~nm}$. This indicates the possibility of dispersion management by modifying the silicon-core geometry, similarly to fibers $[57,58]$. However, as shown in Fig. 2(a), while the first zero-dispersion wavelength is highly dependent on the width, the second one, close to $2300 \mathrm{~nm}$, is much less so. Pumping around $2200 \mathrm{~nm}$, the dispersion map is thus expected to mainly affect the blue side of the broadened spectra. The waveguides considered in this work have either a fixed or a variable width in the range $500-1000 \mathrm{~nm}$ and are all $3 \mathrm{~mm}$ long. The dispersion maps are designed following the same methodology as in our previous work [59]. For the ST waveguide, we impose the taper section to be located between 1 and $2 \mathrm{~mm}$. The DM structure consists of several taper sections between adjustment points (i.e., positions where the slope changes). To limit the size of the parameter space, we restrict ourselves to seven adjustment points, located at $0,0.25,0.5,0.75,1$, 2 , and $3 \mathrm{~mm}$. Their number and location are suggested by the typical dynamics encountered in fixed-width waveguides, in which the fission length is close to $1 \mathrm{~mm}$, for the pulse duration and peak power considered. We stress that this kind of map includes fixed-width waveguides as well as all tapers starting and ending at any adjustment points. The optimum varying-dispersion profile for each structure, i.e., the waveguide width at each adjustment point, is found by resorting to a genetic algorithm with the same fitness function as in Ref. [59], computed in the range 1200-2800 $\mathrm{nm}$. This fitness function produces a trade-off between a broad and a flat output spectrum.

The numerical simulations of the SC generation are performed by solving, with the split-step Fourier method, the generalized nonlinear Schrödinger equation [37], taking into account the full dispersion curve computed using a commercial software package (Lumerical, Inc.), the optical-Kerr and the Raman effects, the three-photon absorption, the free-carrier dispersion and absorption, the avalanche ionization, and the shock term $\left(\tau_{\text {shock }}=\right.$ $\left.1 / \omega_{0}\right)$. The nonlinear coefficients for the reference waveguide width of $800 \mathrm{~nm}$ are $\gamma=220 \mathrm{~W}^{-1} \mathrm{~m}^{-1}$ and $\alpha=$ $0.022 \mathrm{~cm}^{3} \mathrm{GW}^{-2}$ at the pump wavelength $[36,60]$. These coefficients are scaled with the effective mode area at other waveguide widths. The generalized nonlinear Schrödinger equation describes the evolution of the temporal envelope of the electric field. It has been proven to be useful

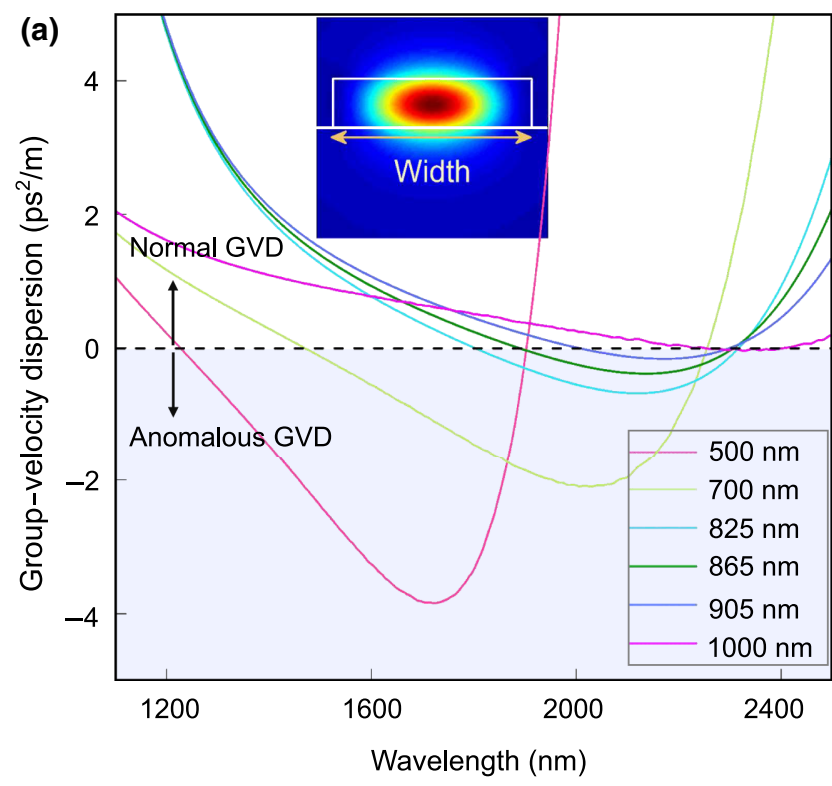

(b)

\begin{tabular}{|c|c|c|c|c|c|c|c|}
\hline$Z(\mathrm{~mm})$ & 0 & 0.25 & 0.5 & 0.75 & 1 & 2 & 3 \\
\hline ST & 870 & 870 & 870 & 870 & 870 & 780 & 780 \\
\hline DM & 550 & 990 & 820 & 880 & 890 & 770 & 810 \\
\hline
\end{tabular}

(c)

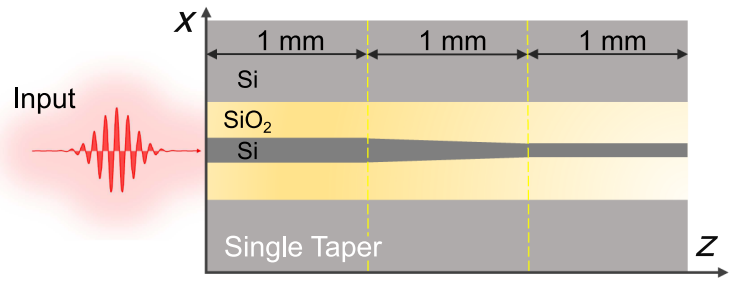

(d)

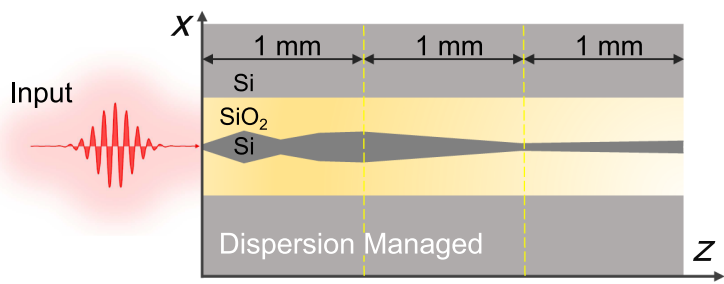

FIG. 2. (a) The computed group-velocity dispersion of the fundamental quasi-TE mode of 220-nm-thick SOI waveguides with fixed widths of 500, 700, 825, 865, 905 and $1000 \mathrm{~nm}$. The inset shows the fundamental transverse mode in the 825-nm-wide waveguide at $2200 \mathrm{~nm}$. (b) The dispersion map of the ST and DM waveguides obtained by the optimization algorithm. The table gives the nominal width at the adjustment points in nanometers as a function of their position $(z)$ along the waveguide. (c),(d) The schematic geometry of the ST and DM structures. 
to simulate the generation of octave-spanning spectra in various contexts (see, e.g., Ref. $[61,62])$. Note that more advanced numerical methods, such as those based on the forward Maxwell equation [63,64], could be used as they are appropriate to simulate the dynamics of broadband waves with complex dispersion and absorption spectra. For the design optimization, we consider a $70-\mathrm{fs} 32-\mathrm{W}-$ peak-power hyperbolic secant input pulse centered at 2200 $\mathrm{nm}$. Applying the optimization algorithm, the simulation quickly converges for the fixed-width waveguide to an optimal width of $865 \mathrm{~nm}$. The optimal dispersion maps for the considered ST and DM structures are given in Fig. 2.

\section{B. Results and discussion}

We start by generating supercontinua in waveguides with nominal fixed widths of $825 \mathrm{~nm}, 865 \mathrm{~nm}$, and $905 \mathrm{~nm}$. These serve as benchmarks to compare with supercontinuum generation (SCG) in varying-dispersion waveguides, as well as for calibrating the dispersion curves. Experimentally, the broadest spectra are obtained at a pump wavelength of $2260 \mathrm{~nm}$ in the FW waveguide but also in the DM and ST ones. The little shift from the design wavelength could be explained by small differences between the computed and the actual dispersion. This wavelength of 2260 $\mathrm{nm}$ will therefore serve as the input-pump wavelength from now on. Measured and simulated output spectra are plotted in Figures 3(a), 3(b), and 3(d).

In the $825-\mathrm{nm}$ FW structure, a distinct dispersive wave can be seen in the normal-dispersion region on the blue side of the pump wavelength [65]. This dispersive wave remains connected to the central part of the spectrum in the $865-\mathrm{nm}$ waveguide, but can barely be seen in the largest one, in agreement with the corresponding dispersion curves [see Fig. 2(a)]. The broadest spectrum is obtained in the 825-nm FW waveguide, for which the resonant dispersive wave is emitted the farthest from the pump wavelength. This results in a spectrum extending from $1168 \mathrm{~nm}$, close to the silicon band edge, up to 2695 $\mathrm{nm}$. The numerical simulations of the SCG from a hyperbolic secant input pulse with 70 fs duration agree well with the experiments, except beyond $2.6 \mu \mathrm{m}$, where a larger spectral density is predicted. This discrepancy is encountered in all waveguides, when the light is collected with the lensed fiber but also with a NIR microscope objective. It is thus attributed to higher losses than expected for large wavelengths, at which the fundamental TE mode is mainly located around the lateral waveguide edges. To take into account the difference between the computed and the actual dispersion properties, we consider the waveguide
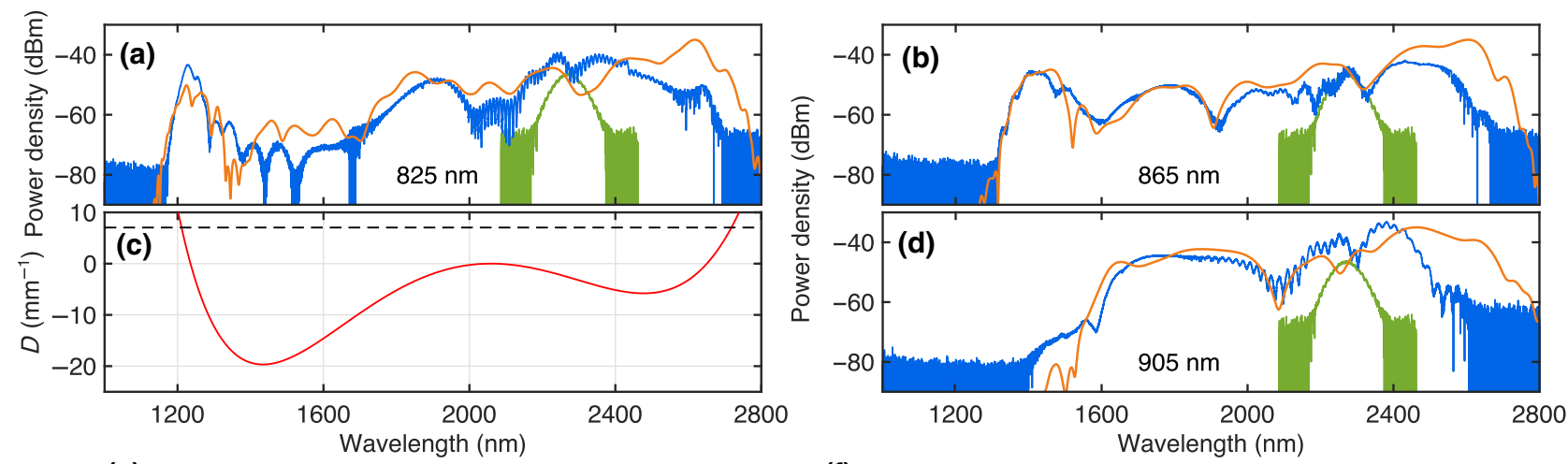

(e)

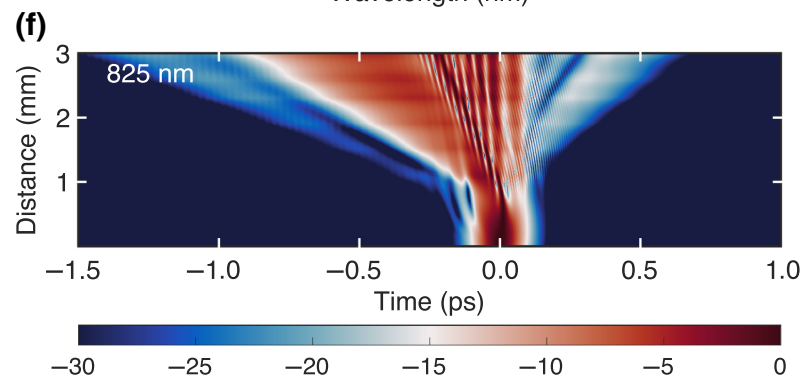

FIG. 3. Experimental (blue) and simulated (orange) output spectra in the waveguides of nominal width (a) $825 \mathrm{~nm}$, (b) $865 \mathrm{~nm}$, and (d) $905 \mathrm{~nm}$ for a pump pulse at $2260 \mathrm{~nm}$ (green). The widths used to compute the dispersion properties differ from the nominal ones. They are 830,880 , and $935 \mathrm{~nm}$, respectively. The experimental average free-space input power is $90 \mathrm{~mW}$. In the simulations, the input peak powers are 54,30, and $40 \mathrm{~W}$, respectively. (c) The wave-number profile $D(\omega)$ of the 825 -nm FW waveguide as used in the simulation, plotted for a 2059-nm wavelength (for further explanation, see the main text). Resonant dispersive waves are emitted at wavelengths satisfying the phase-matching condition given in Eq. (1), i.e., at the crossing points with the horizontal dashed line. (e),(f) The evolution of the simulated spectral and temporal pulse profiles along the $825-\mathrm{nm}$ FW silicon waveguide. The color bars give the normalized power density (e) and the normalized power (f) in decibels. 
width as a free parameter. The widths are set to 830,880 , and $925 \mathrm{~nm}$ in the simulations for the nominal widths of 825,865 and $905 \mathrm{~nm}$, respectively. These values will later be used as a guideline to adjust the width profiles of the ST and the DM waveguides in the simulations. We can finally note that the position of the dispersive wave generated at short wavelengths in the $825-\mathrm{nm}$ waveguide coincides very well with the phase-matching condition [66] (see also Fig. 3):

$$
D\left(\omega_{\mathrm{DW}}\right)=\beta\left(\omega_{\mathrm{DW}}\right)-\beta\left(\omega_{p}\right)-\left(\omega_{\mathrm{DW}}-\omega_{p}\right) / v_{g p} \approx 1 / 2 P_{p} \gamma,
$$

where $\omega_{\mathrm{DW}, p}$ are the frequency of the resonant dispersive wave and of the pump and $v_{g p}$ and $P_{p}$ are the group velocity and the peak power of the pump. The main peak of the temporally focused pulse at the fission point is here considered as the pump and corresponds to a 64-W-peakpower pulse at $2059 \mathrm{~nm}$. This $D(\omega)$ curve also predicts the emission of a second resonant dispersive wave at about $2650 \mathrm{~nm}$, which could well explain the small peak on the right edge of the experimental spectrum. We then consider the propagation in the ST and DM waveguides. In both cases, the spectra are more than one octave wide, extending from $1170 \mathrm{~nm}$ to $2680 \mathrm{~nm}$ in the ST waveguide and from $1190 \mathrm{~nm}$ to $2680 \mathrm{~nm}$ in the DM one [see Figs. 4(a) and 4(b)].

Looking at the output spectra, we can see that the SC generated in the DM waveguide is clearly flatter than in the ST and in the $825-\mathrm{nm} \mathrm{FW}$ ones at similar input power, with at least $10 \mathrm{~dB}$ more spectral density in the (1330-1700)nm-wavelength range. Compared to the FW structure, the main difference in the dynamics of the SCG in the ST and DM structures can be identified by numerical simulations of the spectral and temporal evolutions of the pulse profile. On the one hand, we can readily see the emission of the high-frequency resonant dispersive wave in the 825-nm FW waveguide in the temporal domain (with a positive delay), as it travels slower than the main pulse [see Fig. 3(f)]. On the other hand, this dispersive wave cannot be discerned in the ST one and it emerges only beyond $2.5 \mathrm{~mm}$ in the DM structure [see Figs. 4(e) and 4(f)], indicating that following its emission, this dispersive wave could interact with the main pulse due to the axially varying-dispersion properties. This is confirmed in the spectral domain: between $z \approx 1.5 \mathrm{~mm}$ and $z \approx 2 \mathrm{~mm}$, i.e., in a decreasing taper section of the ST and of the DM waveguides, the dispersive wave, initially emitted around $1400 \mathrm{~nm}$, is frequency shifted toward $1200 \mathrm{~nm}$. Since this frequency shift is more pronounced in the DM waveguide, we only focus on the dynamics in this latter structure.

In order to get a better insight into the interactions involved, we can first look at the spectrogram of the pulse at $z=1.5 \mathrm{~mm}$, where the dispersive wave just emerges in the spectrum. The spectrogram plotted in Fig. 5(a) shows
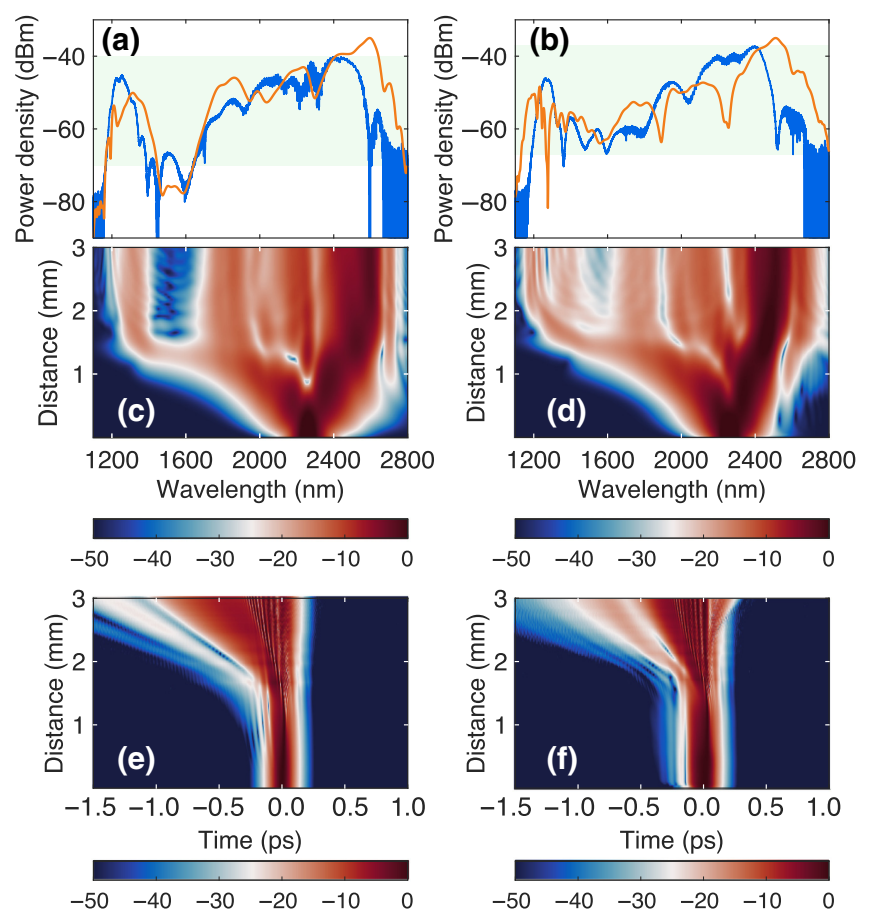

FIG. 4. Supercontinuum generation in the ST (left column) and the DM (right column) waveguides. (a),(b) The recorded (blue) and simulated (orange) spectra. The shaded area highlights a 30-dB dynamic range. The waveguide widths at the adjustment points in the simulations are 885 and $780 \mathrm{~nm}$ for the ST waveguide and $555,1010,825,895,905,775$, and $815 \mathrm{~nm}$ in the DM structure. (c)-(f) The corresponding evolution of the pulse in the spectral and the temporal domain. The average input power measured in free space is $70 \mathrm{~mW}$ in both cases. In the simulations, the input peak power is set to $30 \mathrm{~W}$ for the ST waveguide and to $40 \mathrm{~W}$ for the DM waveguide. The color bars give the normalized power density (c),(d) and the normalized power (e),(f) in decibels.

that the focused pulse consists of a main short pulse, a blue detuned dispersive wave on the trailing edge of the main pulse and, finally, a wave component centered on $2.5 \mu \mathrm{m}$ in the normal-dispersion region and traveling faster than the main pulse (see the Supplemental Material [67]). By numerically filtering the main pulse and the blue detuned dispersive wave, we obtain a 55-W-peakpower 12-fs pulse, centered on $2085 \mathrm{~nm}$ and delayed by 23 fs, and a smaller 5.5-W 20-fs pulse, delayed by 34 fs and centered on $1445 \mathrm{~nm}$. As seen in Fig. 5(d), the simulation of the propagation of two hyperbolic secant short pulses, with these parameters and an arbitrary relative phase, starting at $z=1.5 \mathrm{~mm}$ in the DM waveguide, nicely reproduces the main nonlinear dynamics occurring beyond that location, that is, the continuous blue shift of the dispersive wave from $1445 \mathrm{~nm}$ to $1200 \mathrm{~nm}$. This blue shift can be understood by looking at the dispersion curves $D$ plotted in Fig. 5(b). At $z=1.5 \mathrm{~mm}$, the 1445 $\mathrm{nm}$ wave is actually group-velocity matched with the main 

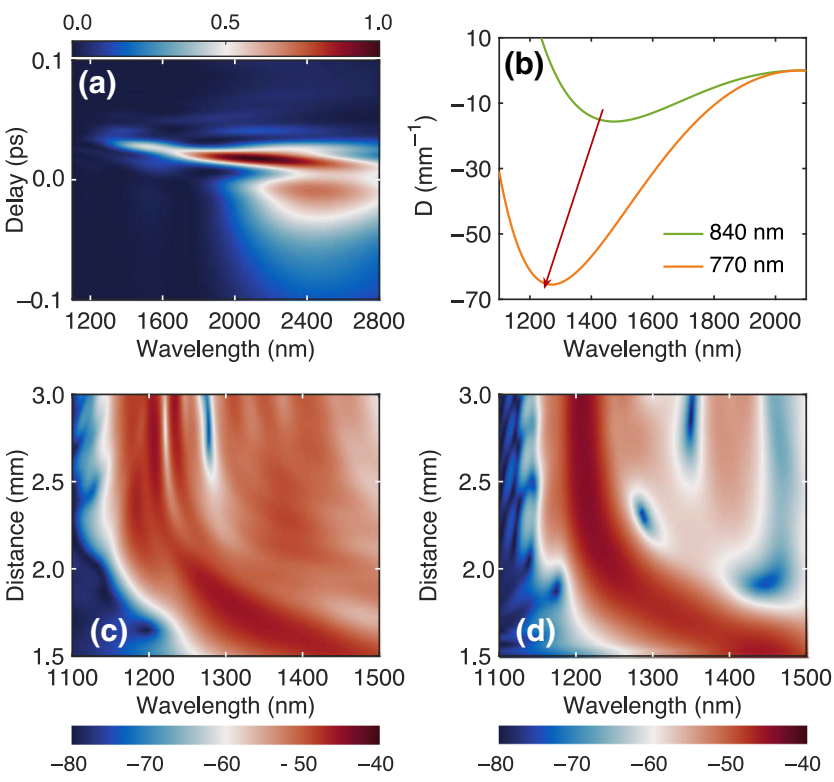

FIG. 5. (a) A spectrogram of the pulse in the DM waveguide at $z=1.5 \mathrm{~mm}$, starting from a $40-\mathrm{W}$-peak-power 70 -fs pulse at $2260 \mathrm{~nm}$. The colors code the normalized power. (b) The dispersion properties (wave number $D$ ) for a reference wavelength of $2085 \mathrm{~nm}$ in 840-nm- and 770-nm-wide waveguides corresponding to the width at $z=1.5 \mathrm{~mm}$ and $z=2 \mathrm{~mm}$ in the DM structure. The arrow schematically shows the evolution of the group-velocity-matched wavelength in the taper section between those two locations. (c),(d) The numerical simulation of the evolution of the spectrum in the DM waveguide between $z=1.5$ $\mathrm{mm}$ and $3 \mathrm{~mm}$ : (c) the same as in Fig. 4(d) and (d) when a 55-W 12-fs pump pulse at $2085 \mathrm{~nm}$, together with a 5.5-W 20-fs seed pulse at $1445 \mathrm{~nm}$, and with an 11-fs relative delay considered at $z=1.5 \mathrm{~mm}$. In this latter case, the spectral shift of the seed pulse is similar to the spectral evolution seen in Fig. 5(c), confirming the trapping mechanism of the dispersive wave encountered in the DM waveguide. The color bars give the normalized power (a), and the normalized power density (c),(d) decibels.

pulse (the same slope). It is thus up-shifted by cross-phase modulation with the trailing edge of the main pulse and slowed down due to the dispersion. However, as the two pulses propagate in the decreasing taper, the up-shifted wave remains group-velocity matched with the main pulse, leading to its continuous blue shift. This nonresonant process is known as intrapulse interaction [68] and refers to the trapping of a dispersive wave by a decelerating soliton, either by Raman scattering [49] or in tapered waveguides [51]. It differs from interpulse interactions, which can also explain the enhanced blue-shifted component of supercontinua observed in tapered waveguides [69,70]. This latter mechanism results, indeed, in a discrete frequency shift due to interactions at event horizon, as observed in fibers [71] or in integrated waveguides [72], and not a continuous frequency shift. Beyond $z=2 \mathrm{~mm}$, the group-velocitymatched wavelength stops decreasing and the nonresonant radiation lags behind the trailing edge of the main pulse, as seen in Fig. 4(f).

Further, we measure, for the DM waveguide, the dependence of the tuning of the input power and wavelength of the pump on the SCG. For potential applications of onchip-generated broad supercontinuum, it would be desirable that their characteristics should be robust, especially due to manufacturing-tolerance issues. Figure 6(a) shows the output spectrum for various input powers corresponding to estimated input-pulse energies ranging between 0.04 and $3.56 \mathrm{pJ}$. At an energy as low as $0.84 \mathrm{pJ}$, the output spectrum is already very broad and smooth, contrary to the one generated in the ST and in the $825-\mathrm{nm}$ FW waveguides [see in Fig. 6(c)], demonstrating the value of the DM design compared to the ST and FW ones. Increasing the input power does not significantly change the output spectrum, probably because the spectrum is limited on the blue side by the absorption of the band gap and, for large wavelengths, by the losses encountered in the waveguide. Since the identified mechanism behind the SCG in the DM structure is not limited to the considered geometry, broader spectra could, in principle, be obtained in silicon waveguides with a larger height and thus better guidance properties beyond $2.8 \mu \mathrm{m}$. We then check the tolerance regarding the input-pump wavelength on the generated SC. The corresponding spectra are displayed in Fig. 6(b) and show that the excellent properties of the supercontinuum are maintained over the full tuning range of our pump laser. All generated spectra at $70 \mathrm{~mW}$ input free-space power (3.12 pJ coupled energy) are octave spanning and extend approximately from $1200 \mathrm{~nm}$ to $2670 \mathrm{~nm}$, as the pump is tuned from $2140 \mathrm{~nm}$ to $2260 \mathrm{~nm}$.

Ideally, the supercontinuum would perform as a broad frequency comb that inherits the spectral coherence from the pump source. We numerically investigate the outputpulse coherence by adding to the input pulse a one-photonper-mode seed field with a randomized phase to each frequency discretization. The first-order coherence $\left|g_{12}\right|$ for 1000 independent pulse pairs is then calculated from the phase and amplitude variations of independent realizations [73]. Figure 6(d) shows that for a 47-W-peak-power 70 -fs input pulse, $\left|g_{12}\right|$ remains above 0.99 over the full bandwidth of the spectrally broadened pulse in the DM waveguide. A very high coherence is thus expected over the whole bandwidth of the supercontinuum. In order to confirm this, we experimentally study the phase coherence in the output spectrum generated in the DM structure. We measure the beat note between the SC and a tunable continuous-wave (cw) laser, an alternative approach to that involving the interference between independent realizations for assessing comb coherence properties [74-76]. The cw source consists of a narrow-band laser (Santec TSL-770, $<60 \mathrm{kHz}$ linewidth), tunable in the 1480-1640 $\mathrm{nm}$ range. This signal is mixed with the supercontinuum by means of a 90:10 fiber coupler (bandwidth 1510-1590 nm) 

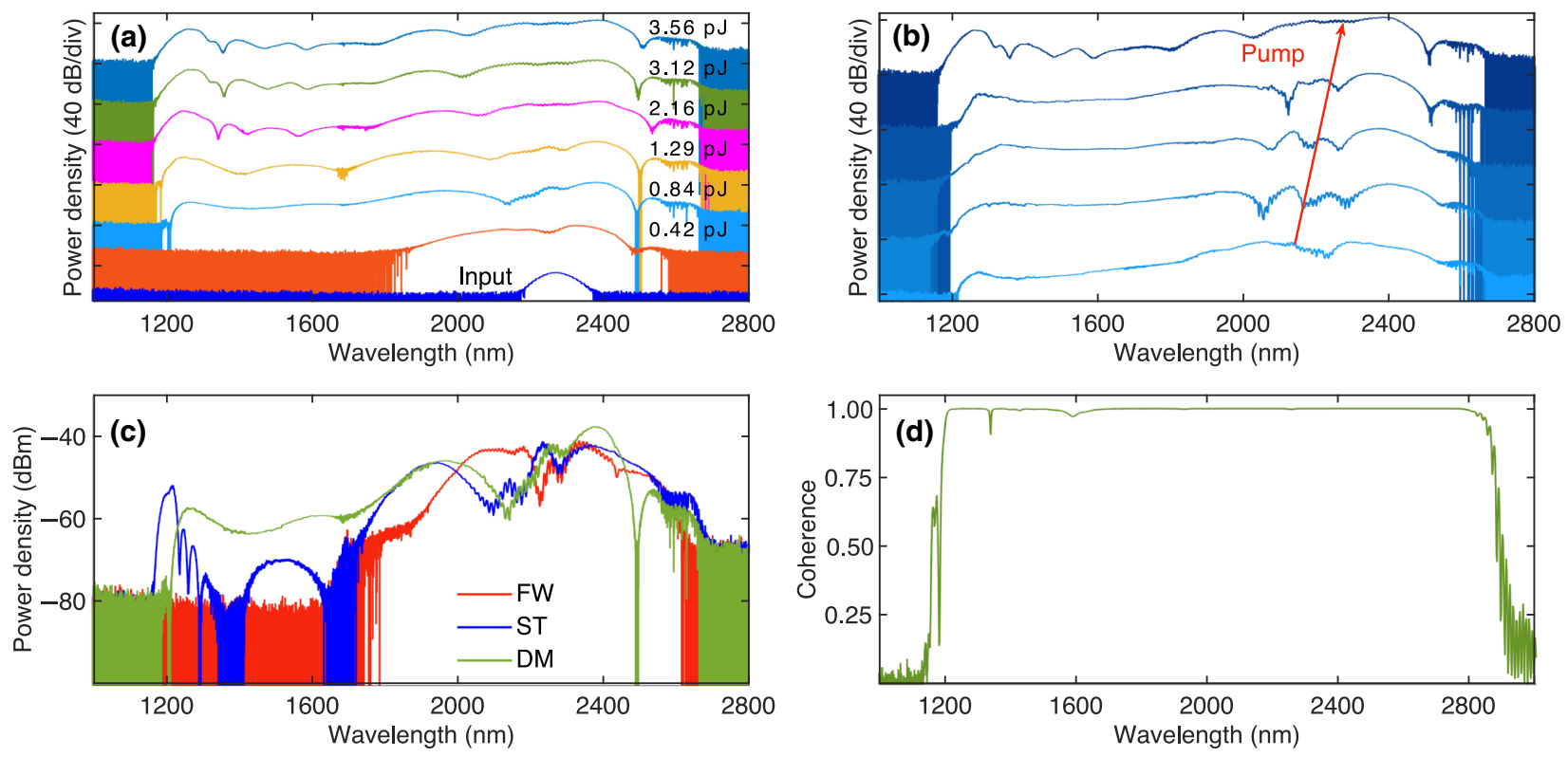

FIG. 6. (a) Experimental output spectra for input-pump-pulse energies between $0.04 \mathrm{pJ}$ (labelled input) and $3.56 \mathrm{pJ}$ in the DM waveguide (bottom to top). (b) The measured output spectra at an input free-space power of $70 \mathrm{~mW}$ (3.12 pJ coupled pulse energy) when the pump is tuned from $2140 \mathrm{~nm}$ to $2260 \mathrm{~nm}$, as indicated by the red arrow. In (a) and (b), the curves are shifted by $40 \mathrm{~dB}$ for clarity. (c) Spectra recorded at the output of the DM (green), ST (blue), and $825 \mathrm{~nm}-\mathrm{FW}$ waveguides (red) for a free space average incident power of $19 \mathrm{~mW}(0.84 \mathrm{pJ}$ coupled energy). (d) The computed degree of first-order coherence of the SCG generated in the DM waveguide at $47 \mathrm{~W}$ input peak power (or, equivalently, $3.7 \mathrm{pJ}$ pulse energy).

and sent to a 125-MHz-bandwidth photodiode, sensitive in the range 900-1700 $\mathrm{nm}$. The rf signal from the photodiode is recorded by a $40 \mathrm{GHz}$ rf spectrum analyzer. The rf spectra measured with the $\mathrm{cw}$ laser at $1520 \mathrm{~nm}$ and $1580 \mathrm{~nm}$ are shown in Figs. 7(a) and 7(b) for the SC plotted in Fig. 4(b). Three isolated lines can be seen, with the strongest one at $82 \mathrm{MHz}$ corresponding to the repetition rate of the pump laser. The two others are the heterodyne beat notes between the two closest lines of the supercontinuum and the cw laser. The spectrum acquired with a $10 \mathrm{kHz}$ resolution shows that the spectral width of the beating signal is about $50 \mathrm{kHz}$ and is thus limited by the linewidth of (a)

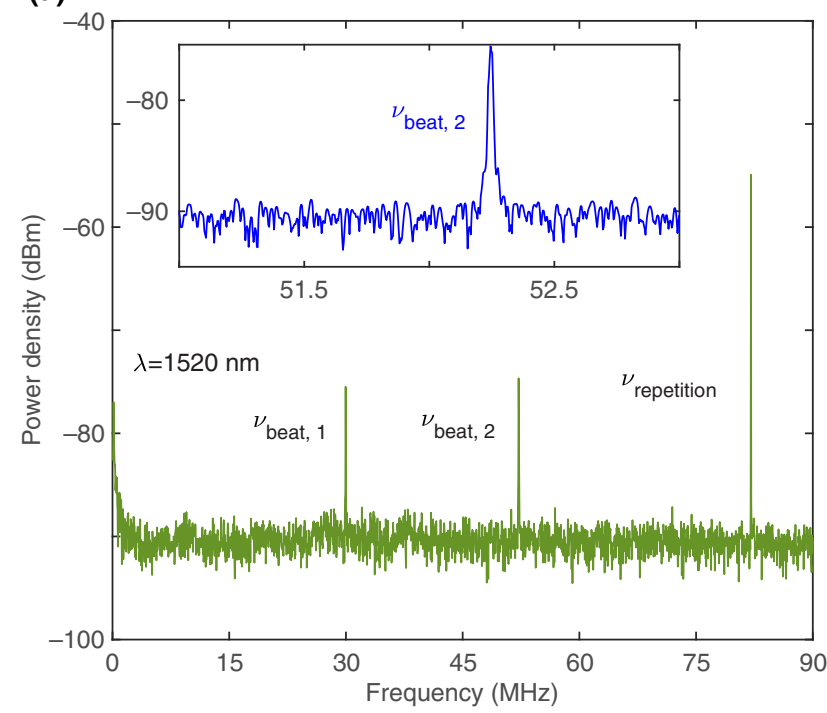

(b)

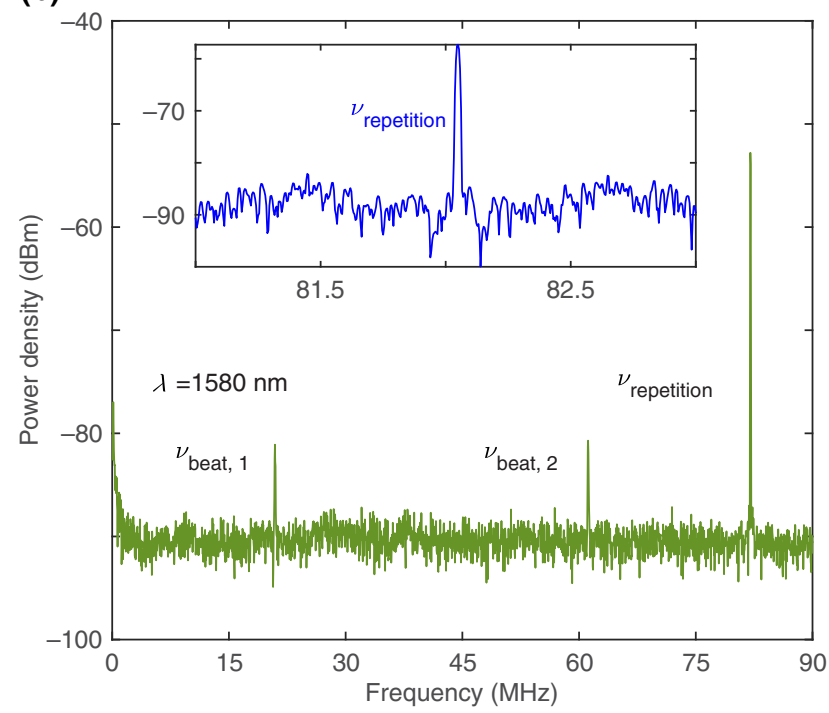

FIG. 7. The beat notes measured at the DM waveguide output with a cw wavelength at (a) $1520 \mathrm{~nm}$ and (b) $1580 \mathrm{~nm}$. The resolution bandwidth is $100 \mathrm{kHz}$. The insets show the spectrum of the beat note measured with a resolution of $10 \mathrm{kHz}$. 
the $\mathrm{cw}$ laser. These results confirm the excellent coherence properties of the broadened frequency comb by supercontinuum generation, in the measured spectral band around $1550 \mathrm{~nm}$. We can finally note that, in this spectral band, only the SC generated in the DM waveguide has a sufficient spectral density to perform beat-note measurements [see Figs. 3(a) and 4(a) and 4(b)]. The low power threshold to get broadband SC in DM structures and the high coherence of the resulting frequency comb is promising for robust chip-scale frequency-comb sources. With the recent demonstration of silicon-based second-harmonic generation [77] and integrated thulium mode-locked laser sources [78], our results could be of benefit, for instance, to the development of fully integrated optical-frequency synthesizers. The generation of a strong dispersive wave far from the pump, which is needed for the comb stabilization of the carrier offset frequency by $f-2 f$ interferometry, could indeed be combined with a flat and smooth coherent spectrum between this latter wavelength and the pump one, as seen in Fig. 6. Compared to Ref. [38], this would extend the wavelength range suitable for optical-frequency synthesis in the range $1.2-1.5 \mu \mathrm{m}$.

\section{CONCLUSION}

In this work, we study the ability of varying-dispersion waveguides to achieve low-power broadband coherent supercontinuum. The design of the best dispersion map to achieve broadband SC is based on numerical simulations of the pulse propagation and an optimization algorithm. Fixed-width and ST structures are also considered. We experimentally demonstrate octave-spanning supercontinuum generation from a sub-100-fs input pulse at $2260 \mathrm{~nm}$, covering the full transmission window of our waveguides. We show that the SC generated in the DM waveguide is as wide as in the ST and fixed-width ones but is flatter and requires less input power. From numerical simulations, we infer that, in the DM waveguide, the dispersive wave on the blue side of the spectrum is first emitted close to the pump wavelength. It is then trapped and continuously blue shifted by the main compressed pulse as they both propagate in a taper section of the DM waveguide. This mechanism enables the efficient generation of the dispersive wave, as it is emitted closer to the pump, and then the spreading of its energy farther away by the trapping mechanism. It thus requires less pump power than in dispersion-engineered fixed-width waveguides to achieve similar spectral broadening. We stress that it is the optimization of the dispersion profile, on the basis of the inputpulse properties, that allows the dispersive wave, once emitted, to be continuously group-velocity matched with the pump and thus trapped and blue shifted. In addition, the output spectrum generated in our DM waveguide is robust against variations in input power (from approximately 20 $\mathrm{W}$ to approximately $80 \mathrm{~W}$ peak) or pump wavelength in the range 2140-2260 $\mathrm{nm}$ and shows excellent coherence properties despite the encountered complex dynamics. This demonstrates the benefit of DM structures over regular fixed-width dispersion-engineered waveguides and establishes them as promising scalable platforms for many applications, such as the next generation of photonic frequency synthesizers [38], high-precision optical clocks $[15,16]$, and spectroscopy devices [12].

\section{ACKNOWLEDGMENTS}

This work was supported by the Fonds de la Recherche Scientifique-FNRS under Grants No. PDR.T.1084.15 and No. PDR.T.1085.18. F.L. acknowledges the support of the Fonds de la Recherche Scientifique-FNRS. B.K. and F.L. acknowledge funding from the European Research Council (ERC) under the European Union's Horizon 2020 research and innovation program (Grant Agreements No. 759483 and No. 757800).

[1] P. A. Franken, A. E. Hill, C. W. Peters, and G. Weinreich, Generation of Optical Harmonics, Phys. Rev. Lett. 7, 118 (1961).

[2] J. M. Dudley and J. R. Taylor, Supercontinuum Generation in Optical Fibers (Cambridge University, Cambridge, 2010), 1 st ed.

[3] R. R. Alfano and S. L. Shapiro, Observation of Self-Phase Modulation and Small-Scale Filaments in Crystals and Glasses, Phys. Rev. Lett. 24, 592 (1970).

[4] P. B. Corkum, C. Rolland, and T. Srinivasan-Rao, Supercontinuum Generation in Gases, Phys. Rev. Lett. 57, 2268 (1986).

[5] V. P. Kandidov, O. G. Kosareva, I. S. Golubtsov, W. Liu, A. Becker, N. Akozbek, C. M. Bowden, and S. L. Chin, Self-transformation of a powerful femtosecond laser pulse into a white-light laser pulse in bulk optical media (or supercontinuum generation), Appl. Phys. B 77, 149 (2003).

[6] J. Bethge, A. Husakou, F. Mitschke, F. Noack, U. Griebner, G. Steinmeyer, and J. Herrmann, Two-octave supercontinuum generation in a water-filled photonic crystal fiber, Opt. Express 18, 6230 (2010).

[7] A. Dubietis, G. Tamosauskas, R. Šuminas, V. Jukna, and A. Couairon, Ultrafast supercontinuum generation in bulk condensed media, Lithuanian J. Phys. 57, 113 (2017).

[8] K. Werner, M. G. Hastings, A. Schweinsberg, B. L. Wilmer, D. Austin, C. M. Wolfe, M. Kolesik, T. R. Ensley, L. Vanderhoef, A. Valenzuela, and E. Chowdhury, Ultrafast mid-infrared high harmonic and supercontinuum generation with $n_{2}$ characterization in zinc selenide, Opt. Express 27, 2867 (2019).

[9] R. Kou, T. Hatakeyama, J. Horng, J.-H. Kang, Y. Wang, $\mathrm{X}$. Zhang, and F. Wang, Mid-IR broadband supercontinuum generation from a suspended silicon waveguide, Opt. Lett. 43, 1387 (2018).

[10] M. Porcel, F. Schepers, J. P. Epping, T. Hellwig, M. Hoekman, R. Heideman, P. J. M. van der Slot, C. J. Lee, R. Schmidt, R. Bratschitsch, C. Fallnich, and K.-J. Boller, 
Two-octave spanning supercontinuum generation in stoichiometric silicon nitride waveguides pumped at telecom wavelengths, Opt. Express 25, 1542 (2017).

[11] C. R. Phillips, C. Langrock, J. S. Pelc, M. M. Fejer, I. Hartl, and M. E. Fermann, Supercontinuum generation in quasiphasematched waveguides, Opt. Express 19, 18754 (2011).

[12] D. Grassani, E. Tagkoudi, H. Guo, C. Herkommer, F. Yang, T. J. Kippenberg, and C. S. Brès, Mid infrared gas spectroscopy using efficient fiber laser driven photonic chip-based supercontinuum, Nat. Commun. 10, 1553 (2019).

[13] K. E. Jahromi, Q. Pan, L. Høgstedt, S. M. M. Friis, A. Khodabakhsh, P. M. Moselund, and F. J. M. Harren, Midinfrared supercontinuum-based upconversion detection for trace gas sensing, Opt. Express 27, 24469 (2019).

[14] K. E. Jahromi, Q. Pan, A. Khodabakhsh, C. Sikkens, P. Assman, S. M. Cristescu, P. M. Moselund, M. Janssens, B. E. Verlinden, and F. J. M. Harren, A broadband mid-infrared trace gas sensor using supercontinuum light source: Applications for real-time quality control for fruit storage, Sensors 19, 2334 (2019).

[15] D. R. Carlson, D. D. Hickstein, A. Lind, J. B. Olson, R. W. Fox, R. C. Brown, A. D. Ludlow, Q. Li, D. Westly, H. Leopardi, T. M. Fortier, K. Srinivasan, S. A. Diddams, and S. B. Papp, Photonic-Chip Supercontinuum with Tailored Spectra for Counting Optical Frequencies, Phys. Rev. Appl. 8, 014027 (2017).

[16] E. S. Lamb, D. R. Carlson, D. D. Hickstein, J. R. Stone, S. A. Diddams, and S. B. Papp, Optical-Frequency Measurements with a Kerr Microcomb and Photonic-Chip Supercontinuum, Phys. Rev. Appl. 9, 024030 (2018).

[17] N. Singh, M. Xin, N. Li, D. Vermeulen, A. Ruocco, E. S. Magden, K. Shtyrkova, P. T. Callahan, C. Baiocco, E. Ippen, F. X. Kärtner, and M. R. Watts, in Conference on Lasers and Electro-Optics (Optical Society of America, San Jose, California United States, 2018), p. SM3L.1.

[18] A. Okamura, Y. Sakakibara, E. Omoda, H. Kataura, and N. Nishizawa, Experimental analysis of coherent supercontinuum generation and ultrashort pulse generation using crosscorrelation frequency resolved optical gating (X-FROG), J. Opt. Soc. Am. B 32, 400 (2015).

[19] H. Saghaei, Supercontinuum source for dense wavelength division multiplexing in square photonic crystal fiber via fluidic infiltration approach, Radioengineering 26, 16 (2017).

[20] H. Tu and S. A. Boppart, Coherent fiber supercontinuum for biophotonics, Laser Photonics Rev. 7, 628 (2013).

[21] A. Bassi, A. Farina, C. D’Andrea, A. Pifferi, G. Valentini, and R. Cubeddu, Portable, large-bandwidth time-resolved system for diffuse optical spectroscopy, Opt. Express 15, 14482 (2007).

[22] G. McConnell, Confocal laser scanning fluorescence microscopy with a visible continuum source, Opt. Express 12, 2844 (2004).

[23] B. Kuyken, X. Liu, R. M. Osgood, R. Baets, G. Roelkens, and W. M. J. Green, Mid-infrared to telecom-band supercontinuum generation in highly nonlinear silicon-oninsulator wire waveguides, Opt. Express 19, 20172 (2011).
[24] D. Y. Oh, D. Sell, H. Lee, K. Y. Yang, S. A. Diddams, and K. J. Vahala, Supercontinuum generation in an on-chip silica waveguide, Opt. Lett. 39, 1046 (2014).

[25] X. Liu, M. Pu, B. Zhou, C. J. Krückel, A. Fülöp, V. TorresCompany, and M. Bache, Octave-spanning supercontinuum generation in a silicon-rich nitride waveguide, Opt. Lett. 41, 2719 (2016).

[26] A. Shams-Ansari, P. Latawiec, Y. Okawachi, V. Venkataraman, M. Yu, B. Desiatov, H. Atikian, G. L. Harris, N. Picqué, A. L. Gaeta, and M. Lončar, Supercontinuum generation in angle-etched diamond waveguides, Opt. Lett. 44, 4056 (2019).

[27] N. Singh, D. D. Hudson, Y. Yu, C. Grillet, S. D. Jackson, A. Casas-Bedoya, A. Read, P. Atanackovic, S. G. Duvall, S. Palomba, B. Luther-Davies, S. Madden, D. J. Moss, and B. J. Eggleton, Midinfrared supercontinuum generation from 2 to $6 \mu \mathrm{m}$ in a silicon nanowire, Optica 2, 797 (2015).

[28] B. Kuyken, M. Billet, F. Leo, K. Yvind, and M. Pu, Octave-spanning coherent supercontinuum generation in an AlGaAs-on-insulator waveguide, Opt. Lett. 45, 603 (2020).

[29] J. Sanghera, I. Aggarwal, L. Shaw, C. Florea, P. Pureza, V. Q. Nguyen, and F. Kung, Nonlinear properties of chalcogenide glass fibers, J. Optolec. Adv. Mater. 8, 2148 (2007).

[30] K. S. Kim, R. H. Stolen, W. A. Reed, and K. W. Quoi, Measurement of the nonlinear index of silica-core and dispersion-shifted fibers, Opt. Lett. 19, 257 (1994).

[31] L. Yin, Q. Lin, and G. P. Agrawal, Soliton fission and supercontinuum generation in silicon waveguides, Opt. Lett. 32, 391 (2007).

[32] I.-W. Hsieh, X. Chen, X. Liu, J. I. Dadap, N. C. Panoiu, C.-Y. Chou, F. Xia, W. M. Green, Y. A. Vlasov, and R. M. Osgood, Supercontinuum generation in silicon photonic wires, Opt. Express 15, 15242 (2007).

[33] F. Leo, S.-P. Gorza, J. Safioui, P. Kockaert, S. Coen, U. Dave, B. Kuyken, and G. Roelkens, Dispersive wave emission and supercontinuum generation in a silicon wire waveguide pumped around the $1550 \mathrm{~nm}$ telecommunication wavelength, Opt. Lett. 39, 3623 (2014).

[34] T. K. Liang and H. K. Tsang, Role of free carriers from two-photon absorption in Raman amplification in siliconon-insulator waveguides, App. Phys. Lett. 84, 2745 (2004).

[35] B. Jalali, Silicon photonics: Nonlinear optics in the midinfrared, Nat. Photonics 4, 506 (2010).

[36] A. D. Bristow, N. Rotenberg, and H. M. van Driel, Twophoton absorption and Kerr coefficients of silicon for 850-2200 nm, Appl. Phys. Lett. 90, 191104 (2007).

[37] R. K. W. Lau, M. R. E. Lamont, A. G. Griffith, Y. Okawachi, M. Lipson, and A. L. Gaeta, Octavespanning mid-infrared supercontinuum generation in silicon nanowaveguides, Opt. Lett. 39, 4518 (2014).

[38] N. Singh, X. Ming, V. Diedrik, S. Katia, L. Nanxi, C. Patrick, M. E. Salih, R. Alfonso, F. Nicholas, B. Christopher, K. Bill, R. Stojan, I. Erich, K. Franz, and M. R. Watts, Octave-spanning coherent supercontinuum generation in silicon on insulator from $1.06 \mu \mathrm{m}$ to beyond $2.4 \mu \mathrm{m}$, Light Sci. App. 7, 17131 (2017).

[39] A. S. Mayer, A. Klenner, A. R. Johnson, K. Luke, M. R. E. Lamont, Y. Okawachi, M. Lipson, A. L. Gaeta, and U. 
Keller, Frequency comb offset detection using supercontinuum generation in silicon nitride waveguides, Opt. Express 23, 15440 (2015).

[40] S. Xing, S. Kharitonov, J. Hu, and C.-S. Brès, Linearly chirped mid-infrared supercontinuum in all-normaldispersion chalcogenide photonic crystal fibers, Opt. Express 26, 19627 (2018).

[41] M. Sinobad, C. Monat, B. Luther-davies, P. Ma, S. Madden, D. J. Moss, A. Mitchell, D. Allioux, R. Orobtchouk, S. Boutami, J.-M. Hartmann, J.- M. Fedeli, and C. Grillet, Mid-infrared octave spanning supercontinuum generation to $8.5 \mu \mathrm{m}$ in silicon-germanium waveguides, Optica 5, 360 (2018).

[42] U. D. Dave, C. Ciret, S.-P. Gorza, S. Combrie, A. De Rossi, F. Raineri, G. Roelkens, and B. Kuyken, Dispersivewave-based octave-spanning supercontinuum generation in In-GaP membrane waveguides on a silicon substrate, Opt. Lett. 40, 3584 (2015).

[43] X. Liu, A. Bruch, J. Lu, Z. Gong, J. Surya, L. Zhang, J. Wang, J. Yan, and H. Tang, Beyond 100 Thz-spanning ultra-violet frequency combs in a non-centrosymmetric crystalline waveguide, Nat. Commun. 10, 2971 (2019).

[44] H. Guo, C. Herkommer, A. Billat, D. Grassani, C. Zhang, M. Pfeiffer, W. Weng, C.-S. Brès, and T. Kippenberg, Mid-infrared frequency comb via coherent dispersive wave generation in silicon nitride nanophotonic waveguides, Nat. Photonics 12, 330 (2018).

[45] M. Yu, B. Desiatov, Y. Okawachi, A. L. Gaeta, and M. Lončar, Coherent two-octave-spanning supercontinuum generation in lithium-niobate waveguides, Opt. Lett. 44, 1222 (2019).

[46] Q. Lin, O. J. Painter, and G. P. Agrawal, Nonlinear optical phenomena in silicon waveguides: Modeling and applications, Opt. Express 15, 16604 (2007).

[47] T. Hori, J. Takayanagi, N. Nishizawa, and T. Goto, Flatly broadened, wideband and low noise supercontinuum generation in highly nonlinear hybrid fiber, Opt. Express 12, 317 (2004).

[48] J. N. Kutz, C. Lyngå, and B. J. Eggleton, Enhanced supercontinuum generation through dispersion management, Opt. Express 13, 3989 (2005).

[49] A. Gorbach and D. Skryabin, Light trapping in gravitylike potentials and expansion of supercontinuum spectra in photonic-crystal fibres, Nat. Photonics 1, 653 (2007).

[50] A. Kudlinski, V. Pureur, G. Bouwmans, and A. Mussot, Experimental investigation of combined four-wave mixing and Raman effect in the normal dispersion regime of a photonic crystal fiber, Opt. Lett. 33, 2488 (2008).

[51] J. C. Travers and J. R. Taylor, Soliton trapping of dispersive waves in tapered optical fibers, Opt. Lett. 34, 115 (2009).

[52] H. Hu, X. Zhang, W. Li, and N. K. Dutta, Simulation of octave spanning mid-infrared supercontinuum generation in dispersion-varying planar waveguides, Appl. Opt. 54, 3448 (2015).

[53] X. Zhang, H. Hu, W. Li, and N. K. Dutta, Mid-infrared supercontinuum generation in tapered $\mathrm{As}_{2} \mathrm{~S}_{3}$ chalcogenide planar waveguide, J. Modern Opt. 63, 1965 (2016).

[54] A. Ishizawa, T. Goto, R. Kou, T. Tsuchizawa, N. Matsuda, K. Hitachi, T. Nishikawa, K. J. Yamada, T. Sogawa, and H. Gotoh, Octave-spanning supercontinuum generation at telecommunications wavelengths in a precisely dispersion- and length-controlled silicon-wire waveguide with a double taper structure, App. Phys. Lett. 111, 021105 (2017).

[55] N. Singh, D. Vermulen, A. Ruocco, N. Li, E. Ippen, F. X. Kärtner, and M. R. Watts, Supercontinuum generation in varying dispersion and birefringent silicon waveguide, Opt. Express 27, 31698 (2019).

[56] D. R. Carlson, P. Hutchison, D. D. Hickstein, and S. B. Papp, Generating few-cycle pulses with integrated nonlinear photonics, Opt. Express 27, 37374 (2019).

[57] W. Q. Zhang, S. Afshar, and T. M. Monro, A genetic algorithm based approach to fiber design for high coherence and large bandwidth supercontinuum generation, Opt. Express 17, 19311 (2009).

[58] F. R. Arteaga-Sierra, C. Milián, I. Torres-Gómez, M. Torres-Cisneros, G. Moltó, and A. Ferrando, Supercontinuum optimization for dual-soliton based light sources using genetic algorithms in a grid platform, Opt. Express 22, 23686 (2014).

[59] C. Ciret and S.-P. Gorza, Generation of ultra-broadband coherent supercontinua in tapered and dispersion-managed silicon nanophotonic waveguides, J. Opt. Soc. Am. B 34, 1156 (2017).

[60] S. Pearl, N. Rotenberg, and H. M. van Driel, Three photon absorption in silicon for 2300-3300 nm, App. Phys. Lett. 93, 131102 (2008).

[61] S. Coen, H. G. Randle, T. Sylvestre, and M. Erkintalo, Modeling of octave-spanning Kerr frequency combs using a generalized mean-field Lugiato-Lefever model, Opt. Lett. 38, 37 (2013).

[62] J. Lu, J. B. Surya, X. Liu, Y. Xu, and H. X. Tang, Octavespanning supercontinuum generation in nanoscale lithium niobate waveguides, Opt. Lett. 44, 1492 (2019).

[63] A. V. Husakou and J. Herrmann, Supercontinuum Generation of Higher-Order Solitons by Fission in Photonic Crystal Fibers, Phys. Rev. Lett. 87, 203901 (2001).

[64] N. A. Panov, D. E. Shipilo, V. A. Andreeva, O. G. Kosareva, A. M. Saletsky, H. Xu, and P. Polynkin, Supercontinuum of a $3.9 \mu \mathrm{m}$ filament in air: Formation of a twooctave plateau and nonlinearly enhanced linear absorption, Phys. Rev. A 94, 041801(R) (2016).

[65] C. Ciret, S.-P. Gorza, C. Husko, G. Roelkens, B. Kuyken, and F. Leo, Physical origin of higher-order soliton fission in nanophotonic semiconductor waveguides, Sci. Rep. 8, 17177 (2018).

[66] D. V. Skryabin and A. V. Yulin, Theory of generation of new frequencies by mixing of solitons and dispersive waves in optical fibers, Phys. Rev. E 72, 016619 (2005).

[67] See the Supplemental Material at http://link.aps.org/supple mental/10.1103/PhysRevApplied.14.054045 showing the evolution of the spectrogram of the pulse with the propagation distance in the dispersion-managed waveguide.

[68] A. V. Gorbach, D. V. Skryabin, J. M. Stone, and J. C. Knight, Four-wave mixing of solitons with radiation and quasi-nondispersive wave packets at the short-wavelength edge of a supercontinuum, Opt. Express 14, 9854 (2006).

[69] A. Bendahmane, A. Mussot, M. Conforti, and A. Kudlinski, Observation of the stepwise blue shift of a dispersive wave 
preceding its trapping by a soliton, Opt. Express 23, 16595 (2015).

[70] S. F. Wang, A. Mussot, M. Conforti, A. Bendahmane, X. L. Zeng, and A. Kudlinski, Optical event horizons from the collision of a soliton and its own dispersive wave, Phys. Rev. A 92, 023837 (2015).

[71] T. G. Philbin, C. Kuklewicz, S. Robertson, S. Hill, F. König, and U. Leonhardt, Fiber-optical analog of the event horizon, Science 319, 1367 (2008).

[72] C. Ciret, F. Leo, B. Kuyken, G. R. Roelkens, and S.-P. Gorza, Observation of an optical event horizon in a siliconon-insulator photonic wire waveguide, Opt. Express 24, 114 (2016).

[73] J. M. Dudley and S. Coen, Numerical simulations and coherence properties of supercontinuum generation in photonic crystal and tapered optical fibers, IEEE J. Sel. Top. Quantum Electron. 8, 651 (2002).
[74] F. Lu and W. H. Knox, Generation of a broadband continuum with high spectral coherence in tapered single-mode optical fibers, Opt. Express 12, 347 (2004).

[75] J. Ye and S. Cundiff, Femtosecond Optical Frequency Comb: Principle, Operation and Applications (Springer, USA, 2005).

[76] F. Leo, S.-P. Gorza, S. Coen, B. Kuyken, and G. Roelkens, Coherent supercontinuum generation in a silicon photonic wire in the telecommunication wavelength range, Opt. Lett. 40, 123 (2015).

[77] N. Singh, M. Raval, A. Ruocco, and M. R. Watts, Broadband 200-nm second-harmonic generation in silicon in the telecom band, Light Sci. Appl. 9, 17 (2020).

[78] N. Li, P. Purnawirman, Z. Su, E. S. Magden, P. T. Callahan, K. Shtyrkova, M. Xin, A. Ruocco, C. Baiocco, E. P. Ippen, F. X. Kärtner, J. D. B. Bradley, D. Vermeulen, and M. R. Watts, High-power thulium lasers on a silicon photonics platform, Opt. Lett. 42, 1181 (2017). 